\title{
MODULATION OF GASTROINTESTINAL TRANSIT TIME OF SALBUTAMOL SULPHATE BY FLOATING APPROCHES
}

\author{
Verma Pooja*, Roy Choudhary Santanu \\ Sri Sai College Of Pharmacy, Badhani, Pathankot, Punjab \\ *Corresponding Author's Email: verma_sweety786@ymail.com
}

\begin{abstract}
:
The present investigation concerns with the development and evaluation of the floating matrix tablets of Salbutamol Sulphate. Tablets were prepared by wet granulation method. Hydroxypropyl methylcellulose and ethyl cellulose was used as a release retardant material. Sodium bicarbonate and Citric acid was incorporated as a gas-generating agent. The effects of Sodium bicarbonate and citric acid on drug release profile, floating properties and matrix integrity of tablet were investigated. Addition of Citric acid in excess amount results in disintegration of the tablet so the floating tablets are prepared by full $3^{2}$ factorial designs by reducing the ratio of citric acid. Developed formulations were evaluated for their physical characteristics, drug content, floating lag time, floating duration, in vitro drug release profile etc. On the basis of various physical characteristics parameters it was found that all the formulations shows good result. On comparative kinetic modeling study such as (Zero order, Higuchi model and Korsmeyer-Peppas) it was found that all the formulations follow Higuchi model and correlation coefficient $\left(\mathrm{R}^{2}\right)$ and Korsmeyer model values were nearer to unity. Among those formulations F8 and F9 showed $\mathrm{R}^{2}$ value of Higuchi model more near as compared to the other formulation.

Keywords: Salbutamol sulphate, Ethyl cellulose, HPMC, Sodium bicarbonate, Citric acid, Tartaric acid.
\end{abstract}

\section{INTRODUCTION:}

Oral controlled release drug delivery have recently been of increasing interest in pharmaceutical field to achieve improved therapeutic advantages, such as ease of dosing administration, patient compliance and flexibility in formulation $^{(1,2)}$. Drugs with short half-lives and drugs that easily absorbed from gastrointestinal tract (GIT) are eliminated quickly from the systemic circulation. For these types of drugs the development of oral sustainedcontrolled release formulations is an attempt to release the drug slowly into the gastrointestinal tract (GIT) and maintain an effective drug concentration in the systemic circulation for a long time ${ }^{(3,2)}$. But oral sustained drug delivery formulations show some limitations connected with the gastric emptying time; variable and too rapid gastrointestinal transit could result in incomplete drug release from the device into the absorption window leading to diminished efficacy of the administered dose ${ }^{(4,2)}$. Floating drug delivery system is an approach to prolong gastric residence time, thereby targeting site-specific drug release in the upper gastrointestinal tract (GIT) for local or systemic effects (4). In the present investigation, Salbutamol sulfate was selected as a model for the development of gartroretentive drug delivery system (GRFDDS). Salbutamol sulphate act as an $b_{2}$ adrenergic receptor agonist widely used for the treatment of bronchial asthma, chronic bronchitis and emphysema ${ }^{(5)}$. The drug undergoes extensive first-pass metabolism and thus requires frequent administrations by oral route (6). Salbutamol sulphate has a site-specific absorption in stomach and upper part of small intestine ${ }^{(7)}$. Reported oral bioavailability of salbutamol sulphate is $~ 40 \%$; due to extensive metabolism via intestinal sulphonation, first pass metabolism in liver $\&$ also degradation in colon ${ }^{(8)}$. The metabolism is due to extensive sulphonation in gut as compared to liver. The half life of Salbutamol sulphate is about $4.5 \mathrm{hrs}{ }^{(9)}$. A gastroretentive drug delivery system may be advantageous over conventional oral dosage forms and inhalers due to its ability to maintain prolonged therapeutic concentrations in the systemic circulation ${ }^{(10)}$.

\section{MATERIALS AND METHODS}

\section{Materials:}

The drug Salbutamol sulphate was supplied as a gift sample by Ultra Lab Baddi (Himachal Pradesh). All other reagents and chemicals were of analytical grade.

\section{Preparation of floating tablet of Salbutamol sulphate:}

Floating Salbutamol sulphate tablet shall be prepared by using full $3^{2}$ factorial design by varying the ratio of sodium bicarbonate and citric acid which was used as a gas generating agent. All tablets were prepared by wet granulation method. All the polymer and active ingredients were passed through sieve no. 80 separately. Required quantity of Salbutamol sulphate, polymers, gas generating, and diluents were mixed thoroughly in a glass motor pestle and sufficient quantity of binding agent was added slowly to get dough mass. The mass was sieved through no. 8 mesh and dried at $50^{\circ} \mathrm{C}$ temperature for $1 \mathrm{hrs}$. The dried granules retained on 35 no. mesh were mixed with $10 \%$ fines, $2 \%$ talc and $1 \%$ magnesium stearate and the tablet were punched with the help of automatic tablet punching machine with desired hardness, shape and size. The formulation parameters are listed below in table no. 1 .

A Full $3^{2}$ factorial design were made by varying the ratio of gas generating agent: 
Table 1: A Full $3^{2}$ Factorial Design of formulation F1 TO F9:

\begin{tabular}{|c|c|c|c|c|c|c|c|c|}
\hline $\begin{array}{c}\text { Formulation } \\
\text { code }\end{array}$ & Drug & EC & HPMC & NaHCo3 & Citric Acid & Lactose & $\begin{array}{c}\text { Mg. } \\
\text { Sterate } \\
(1 \% \mathrm{w} / \mathrm{w})\end{array}$ & $\begin{array}{c}\text { Talc } \\
(2 \% \mathrm{w} / \mathrm{w})\end{array}$ \\
\hline $\mathrm{F} 1$ & $12 \mathrm{mg}$ & $40 \mathrm{mg}$ & $30 \mathrm{mg}$ & $12 \% \mathrm{w} / \mathrm{w}=12 \mathrm{mg}$ & $0 \% \mathrm{w} / \mathrm{w}=0 \mathrm{mg}$ & $3 \mathrm{mg}$ & $1 \mathrm{mg}$ & $2 \mathrm{mg}$ \\
\hline F2 & $12 \mathrm{mg}$ & $40 \mathrm{mg}$ & $30 \mathrm{mg}$ & $10 \% \mathrm{w} / \mathrm{w}=10 \mathrm{mg}$ & $0 \% \mathrm{w} / \mathrm{w}=0 \mathrm{mg}$ & $5 \mathrm{mg}$ & $1 \mathrm{mg}$ & $2 \mathrm{mg}$ \\
\hline F3 & $12 \mathrm{mg}$ & $40 \mathrm{mg}$ & $30 \mathrm{mg}$ & $8 \% \mathrm{w} / \mathrm{w}=8 \mathrm{mg}$ & $0 \% \mathrm{w} / \mathrm{w}=0 \mathrm{mg}$ & $7 \mathrm{mg}$ & $1 \mathrm{mg}$ & $2 \mathrm{mg}$ \\
\hline F4 & $12 \mathrm{mg}$ & $40 \mathrm{mg}$ & $30 \mathrm{mg}$ & $12 \% \mathrm{w} / \mathrm{w}=12 \mathrm{mg}$ & $0.5 \% \mathrm{w} / \mathrm{w}=0.5 \mathrm{mg}$ & $2.5 \mathrm{mg}$ & $1 \mathrm{mg}$ & $2 \mathrm{mg}$ \\
\hline F5 & $12 \mathrm{mg}$ & $40 \mathrm{mg}$ & $30 \mathrm{mg}$ & $10 \% \mathrm{w} / \mathrm{w}=10 \mathrm{mg}$ & $0.5 \% \mathrm{w} / \mathrm{w}=0.5 \mathrm{mg}$ & $4.5 \mathrm{mg}$ & $1 \mathrm{mg}$ & $2 \mathrm{mg}$ \\
\hline F6 & $12 \mathrm{mg}$ & $40 \mathrm{mg}$ & $30 \mathrm{mg}$ & $8 \% \mathrm{w} / \mathrm{w}=8 \mathrm{mg}$ & $0.5 \% \mathrm{w} / \mathrm{w}=0.5 \mathrm{mg}$ & $6.5 \mathrm{mg}$ & $1 \mathrm{mg}$ & $2 \mathrm{mg}$ \\
\hline F7 & $12 \mathrm{mg}$ & $40 \mathrm{mg}$ & $30 \mathrm{mg}$ & $12 \% \mathrm{w} / \mathrm{w}=12 \mathrm{mg}$ & $1 \% \mathrm{w} / \mathrm{w}=1 \mathrm{mg}$ & $2 \mathrm{mg}$ & $1 \mathrm{mg}$ & $2 \mathrm{mg}$ \\
\hline F8 & $12 \mathrm{mg}$ & $40 \mathrm{mg}$ & $30 \mathrm{mg}$ & $10 \% \mathrm{w} / \mathrm{w}=10 \mathrm{mg}$ & $1 \% \mathrm{w} / \mathrm{w}=1 \mathrm{mg}$ & $4 \mathrm{mg}$ & $1 \mathrm{mg}$ & $2 \mathrm{mg}$ \\
\hline F9 & $12 \mathrm{mg}$ & $40 \mathrm{mg}$ & $30 \mathrm{mg}$ & $8 \% \mathrm{w} / \mathrm{w}=8 \mathrm{mg}$ & $1 \% \mathrm{w} / \mathrm{w}=1 \mathrm{mg}$ & $6 \mathrm{mg}$ & $1 \mathrm{mg}$ & $2 \mathrm{mg}$ \\
\hline
\end{tabular}

\section{EVALUATION PARAMETERS:}

A) Standard calibration curve of salbutamol sulphate in 0.1(N) HCL pH (1.2), and 0.2 (M) Phosphate buffer pH 6.8 was prepared: These standard graph are shown below in result and discussion chapter.

B) Evaluation of Granules: The following evaluation parameters of granules were determined such as Angle of repose, Apparent bulk density, Tapped density, Compressibility index, Hausner's ratio. The results are shown below in table no. 2 .

C) Evaluation Of Salbutamol Sulphate Floating Tablet: These following evaluation parameters of salbutamol sulphate tablet was determined thourghly during my research work i.e General appearance, Thickness, Hardness, Weight variation, Friability, Drug Content, Swelling studies, Buoyancy lag time determination and Total floating time. The results of these various parameters are listed below in table no. 3 .

In-vitro dissolution study:
In vitro drug release studies for the prepared floating tablets of salbutamol sulphate were conducted for a period of $10 \mathrm{hrs}$ with an half of interval USP XXIV type-II (Paddle) dissolution apparatus at $37 \pm 0.5^{\circ} \mathrm{C}$ at $100 \mathrm{rpm}$ using $900 \mathrm{ml}$ of $0.1 \mathrm{~N} \mathrm{HCl}$ as dissolution medium for first two hrs and similarly for next seven hrs dissolution was conducted with $0.2 \mathrm{M}$ Phosphate buffer solution at a predetermined interval of time, $5 \mathrm{ml}$ of sample was withdrawn from the dissolution medium and replaced with fresh medium to maintain the sink condition. After filtration and appropriate dilution, the samples were analyzed for salbutamol sulphate by UV spectrophotometer at $276 \mathrm{~nm}$. Then the release kinetics of the drug salbutamol suphate was studied with the help of percentage cumulative drug release by using the models of release kinetics; such as Zero order release kinetics, First order release kinetics, Higuchi model and KorsmeyerPeppas model.

\section{RESULT AND DISCUSSION:}

A) Standard plot of salbutamol sulphate in 0.1 (N) HCL And 0.2 (M) Phosphate buffer solution:

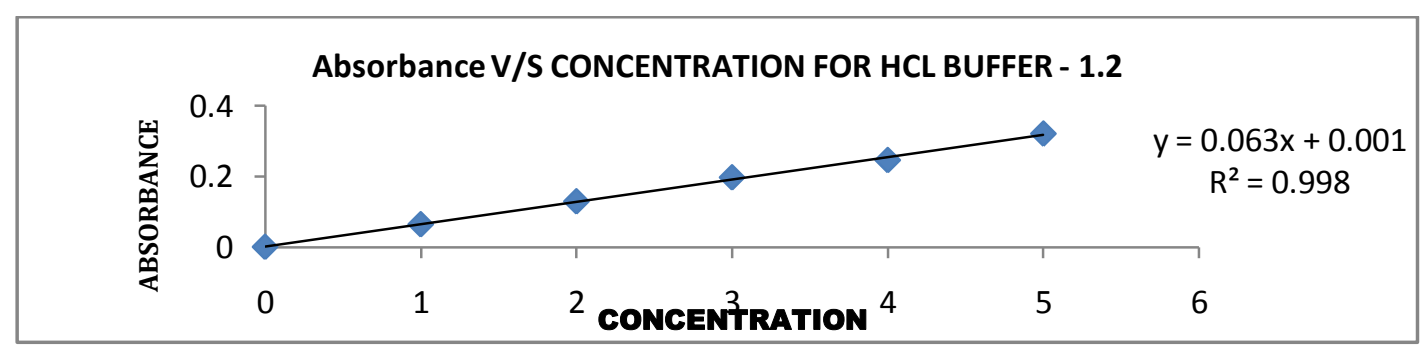

Graph no. 1: Standard plot of Salbutamol sulphate in 0.1 N HCL buffer solution:

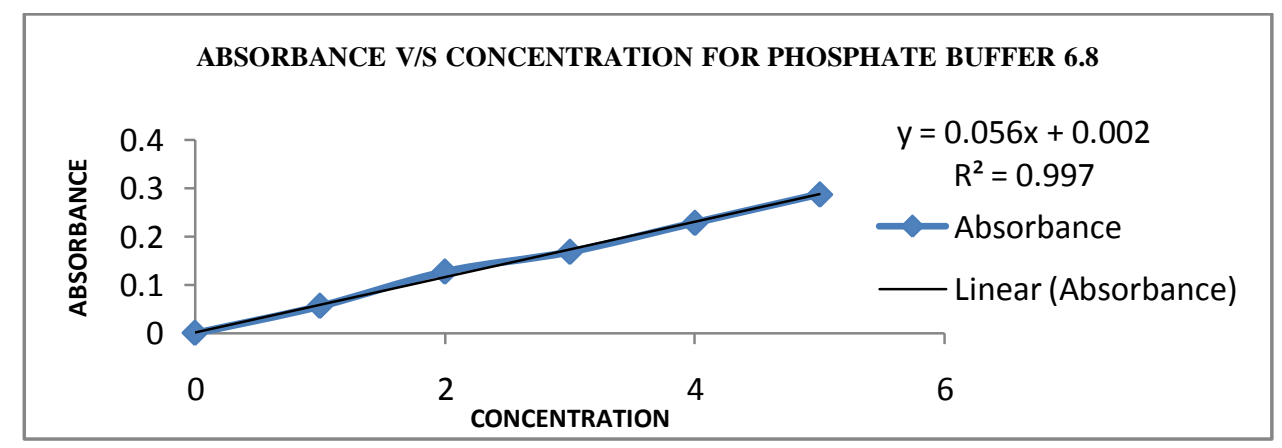

Graph no. 2: Standard plot of salbutamol sulphate in Phosphate buffer solution: 
B) Evaluation of Granules: Evaluation parameters of granules are listed below in table no. 2:

Table no. 2: Shows various Evaluation parameters of granules:

\begin{tabular}{|c|l|l|l|l|l|}
\hline $\begin{array}{c}\text { Formulation } \\
\text { Code }\end{array}$ & $\begin{array}{c}\text { Bulk density (g/cc) } \\
\pm \mathrm{sd}, \mathrm{n}=3\end{array}$ & $\begin{array}{c}\text { Tapped density } \\
(\mathrm{g} / \mathrm{cc}) \pm \mathrm{sd}, \mathrm{n}=3\end{array}$ & $\begin{array}{c}\text { Angle of repose } \\
(\mathrm{degree}) \pm \mathrm{sd}, \mathrm{n}=3\end{array}$ & $\begin{array}{c}\text { Carr's Index (\%) } \pm \\
\mathrm{sd}, \mathrm{n}=3\end{array}$ & $\begin{array}{c}\text { Hausner's } \\
\text { Ratio } \pm \mathrm{sd}, \mathrm{n}=3\end{array}$ \\
\hline F1 & $2.26 \pm 0.20$ & $6.47 \pm 8.85$ & $29.41 \pm 0.4761$ & $8.77 \pm 0.549$ & $1.266 \pm 0.169$ \\
\hline F2 & $0.182 \pm 0.0017$ & $0.23 \pm 0.02$ & $27.28 \pm 0.631$ & $9.77 \pm 0.207$ & $1.373 \pm 0.372$ \\
\hline F3 & $0.435 \pm 0.015$ & $0.44 \pm 0.016$ & $26.133 \pm 0.507$ & $9.12 \pm 0.677$ & $1.11 \pm 0.008$ \\
\hline F4 & $0.28 \pm 0.0020$ & $0.332 \pm 0.0020$ & $30.126 \pm 0.843$ & $14.74 \pm 0.282$ & $1.16 \pm 0.0163$ \\
\hline F5 & $152.6 \pm 2.05$ & $0.164 \pm 0.00163$ & $23.22 \pm 1.077$ & $7.05 \pm 0.658$ & $1.08 \pm 0.016$ \\
\hline F6 & $0.25 \pm 0.016$ & $0.282 \pm 1.632$ & $29.60 \pm 0.656$ & $10.75 \pm 0.449$ & $1.12 \pm 0.016$ \\
\hline F7 & $0.25 \pm 0.81$ & $0.266 \pm 0.0016$ & $26.31 \pm 0.471$ & $6.063 \pm 0.711$ & $1.167 \pm 0.002$ \\
\hline F8 & $0.002 \pm 0.141$ & $0.333 \pm 1.632$ & $29.06 \pm 0.610$ & $14.20 \pm 0.744$ & $1.167 \pm 0.002$ \\
\hline F9 & $0.200 \pm 0.169$ & $7.83 \pm 10.7$ & $27.7 \pm 0.423$ & $20.146 \pm 0.73$ & $1.393 \pm 0.188$ \\
\hline
\end{tabular}

C) Evaluation of tablet: Evaluation parameters of tablet are listed below in table no. 3:

Table 3: Evaluation of various parameters of all formulation of prepared tablet

\begin{tabular}{|c|c|c|c|c|c|c|c|c|c|c|}
\hline \multirow[t]{2}{*}{$\begin{array}{l}\text { Formulation } \\
\text { code }\end{array}$} & \multirow{2}{*}{$\begin{array}{l}\text { Weight } \\
\text { Variation } \\
(\mathrm{mg})\end{array}$} & \multirow[t]{2}{*}{$\begin{array}{l}\text { Diameter } \\
(\mathbf{c m})\end{array}$} & \multirow[t]{2}{*}{$\begin{array}{l}\text { Thickness } \\
\text { (cm) }\end{array}$} & \multirow[t]{2}{*}{$\begin{array}{r}\text { Hardness } \\
(\mathrm{kg} / \mathrm{cm} 2)\end{array}$} & \multirow[t]{2}{*}{$\begin{array}{c}\text { Friability } \\
(\%)\end{array}$} & \multicolumn{2}{|c|}{$\begin{array}{c}\text { Drug }(\%) \\
\text { content }\end{array}$} & \multirow[t]{2}{*}{$\begin{array}{l}\text { Swelling } \\
\text { index }\end{array}$} & \multirow{2}{*}{$\begin{array}{l}\text { Lag } \\
\text { Time } \\
\text { (sec) }\end{array}$} & \multirow{2}{*}{$\begin{array}{l}\text { Total } \\
\text { Floating } \\
\text { Time(hr) }\end{array}$} \\
\hline & & & & & & $1.2 \mathrm{pH}$ & $6.8 \mathrm{pH}$ & & & \\
\hline F1 & $100.4 \pm 4.4$ & $8.00 \pm 0.017$ & $3.05 \pm 0.064$ & $2.63 \pm 0.124$ & $0.8 \pm 0.008$ & 97.83 & 97.08 & 56 & 105 & 12 \\
\hline F2 & $105.6 \pm 5.0$ & $799 \pm 0.006$ & $3.22 \pm 0.085$ & $3.03 \pm 0.124$ & $0.81 \pm 0.016$ & 96.6 & 97.01 & 65 & 85 & 9 \\
\hline F3 & $99.36 \pm 2.9$ & $7.96 \pm 0.001$ & $3.17 \pm 0.110$ & $3.23 \pm 0.205$ & $0.85 \pm 0.028$ & 97.37 & 96.60 & 68 & 45 & 10 \\
\hline F4 & $101.6 \pm 3.0$ & $7.99 \pm 0.019$ & $2.95 \pm 0.056$ & $3.5 \pm 0.163$ & $0.81 \pm 0.001$ & 97.33 & 97.01 & 90 & 150 & 15 \\
\hline F5 & $97.33 \pm 3.0$ & $7.99 \pm 0.016$ & $3.13 \pm 0.067$ & $3.16 \pm 0.205$ & $0.81 \pm 0.016$ & 98.43 & 97.45 & 68 & 80 & 12 \\
\hline F6 & $101.4 \pm 3.9$ & $7.98 \pm 0.039$ & $3.02 \pm 0.124$ & $3.3 \pm 0.163$ & $0.81 \pm 0.008$ & 97.54 & 96.50 & 62 & 60 & 14 \\
\hline F7 & $96.8 \pm 3.2$ & $7.97 \pm 0.017$ & $3.02 \pm 0.124$ & $3.2 \pm 0.163$ & $0.80 \pm 0.005$ & 95.06 & 96.01 & 77 & 180 & 14 \\
\hline F8 & $105.4 \pm 3.1$ & $7.96 \pm 0.049$ & $2.99 \pm 0.085$ & $2.8 \pm 0.163$ & $0.81 \pm 0.008$ & 97.31 & 96.50 & 82 & 40 & 19 \\
\hline F9 & $100.8 \pm 2.8$ & $7.99 \pm 0.021$ & $3.00 \pm 0.061$ & $2.3 \pm 0.163$ & $0.81 \pm 0.008$ & 97.31 & 98.08 & 91 & 80 & 22 \\
\hline
\end{tabular}

Table 4: shows In-vitro Dissolution Profile of Formulation F1 TO F9 in HCL buffer Ph 1.2 I.P for (1-2hr)and Phosphate buffer p.H 6.8 I.P for (10 hrs)

\begin{tabular}{|c|c|c|c|c|c|c|c|c|c|c|}
\hline Time & Time & $\begin{array}{c}\text { \% CAR } \\
\text { F1 }\end{array}$ & $\begin{array}{c}\text { \% CAR } \\
\text { F2 }\end{array}$ & $\begin{array}{c}\text { \% CAR } \\
\text { F3 }\end{array}$ & $\begin{array}{c}\text { \% CAR } \\
\text { F4 }\end{array}$ & $\begin{array}{c}\text { \% CAR } \\
\text { F5 }\end{array}$ & $\begin{array}{c}\text { \% CAR } \\
\text { F6 }\end{array}$ & $\begin{array}{c}\text { \% CAR } \\
\text { F7 }\end{array}$ & $\begin{array}{c}\text { \% CAR } \\
\text { F8 }\end{array}$ & $\begin{array}{c}\text { \% CAR } \\
\text { F9 }\end{array}$ \\
\hline 0 & 0 & 0 & 0 & 0 & 0 & 0 & 0 & 0 & 0 & 0 \\
\hline 0.5 & 0.707 & 11.5 & 12.57 & 13.86 & 18.95 & 20.87 & 7.84 & 8.52 & 1.85 & 1.23 \\
\hline 1 & 1 & 18.3 & 18.08 & 21.8 & 21.84 & 25.56 & 11.26 & 11.09 & 5.25 & 4.78 \\
\hline 1.5 & 1.225 & 22.18 & 21.52 & 28.62 & 25.55 & 28.33 & 15.74 & 13.31 & 8.04 & 8.04 \\
\hline 2 & 1.414 & 30.18 & 22.17 & 31.27 & 27.42 & 33.83 & 21.92 & 15.37 & 14.22 & 13.3 \\
\hline 2.5 & 1.581 & 39.54 & 37.01 & 48.98 & 39.35 & 52.48 & 39.18 & 21.29 & 20.79 & 20.79 \\
\hline 3 & 1.732 & 48.06 & 41.57 & 53.14 & 43.12 & 56.06 & 41.12 & 23.78 & 26.01 & 25.08 \\
\hline 3.5 & 1.871 & 51.41 & 49.6 & 55.75 & 43.88 & 59.92 & 49.43 & 28.53 & 29 & 30.84 \\
\hline 4 & 2 & 52.72 & 55.99 & 57.61 & 49.8 & 63.23 & 52.87 & 34.05 & 33.46 & 34.2 \\
\hline 4.5 & 2.121 & 56.6 & 62.17 & 57.62 & 53.9 & 68.38 & 56.42 & 38.05 & 38.84 & 38.48 \\
\hline 5 & 2.236 & 59.19 & 62.57 & 57.99 & 57.99 & 69.5 & 57.21 & 40.35 & 42.76 & 38.87 \\
\hline 5.5 & 2.345 & 60.3 & 67.05 & 59.84 & 60.61 & 69.88 & 58.44 & 41.89 & 47.96 & 46.09 \\
\hline 6 & 2.449 & 60.68 & 71.17 & 67.99 & 64.87 & 71.15 & 60.29 & 45.3 & 51.5 & 51.5 \\
\hline 6.5 & 2.549 & 68.41 & 74.18 & 73.95 & 65.08 & 71.72 & 61.23 & 47.59 & 56.33 & 56.15 \\
\hline 7 & 2.646 & 72.87 & 79.04 & 74.85 & 65.45 & 75.74 & 61.54 & 48.55 & 62.83 & 60.25 \\
\hline 7.5 & 2.739 & 78.97 & 80.18 & 76.57 & 70.26 & 78.32 & 63.39 & 52.71 & 70.08 & 62.11 \\
\hline 8 & 2.828 & 79.55 & 81.68 & 77.32 & 73.43 & 82.36 & 67.09 & 54.63 & 75.11 & 63.23 \\
\hline 8.5 & 2.915 & 80.66 & 82.25 & 78.06 & 73.64 & 84.03 & 71.72 & 60.9 & 79.58 & 78.03 \\
\hline 9 & 3 & 81.4 & 82.44 & 81.58 & 87.88 & 84.59 & 74.98 & 65.29 & 83.49 & 80.53 \\
\hline 9.5 & 3.082 & 86.56 & 84.86 & 84.19 & 89.62 & 85.13 & 75.76 & 73.45 & 87.4 & 84.24 \\
\hline 10 & 3.162 & 89.71 & 88.41 & 87.9 & 91.29 & 90.07 & 76.7 & 79.56 & 90.38 & 88.7 \\
\hline 10.5 & 3.24 & 91.2 & 91.23 & 88.84 & 91.86 & 91.56 & 79.47 & 83.57 & 91.5 & 89.28 \\
\hline 11 & 3.317 & 91.4 & 92.18 & 92.18 & 92.6 & 91.94 & 80.06 & 87.56 & 94.28 & 89.65 \\
\hline 11.5 & 3.391 & 93.42 & 93.11 & 93.86 & 92.98 & 93.77 & 81.48 & 90.8 & 95.41 & 92.24 \\
\hline 12 & 3.464 & 94.72 & 94.98 & 95.35 & 93.9 & 95.06 & 81.96 & 93.28 & 96.53 & 95.4 \\
\hline
\end{tabular}




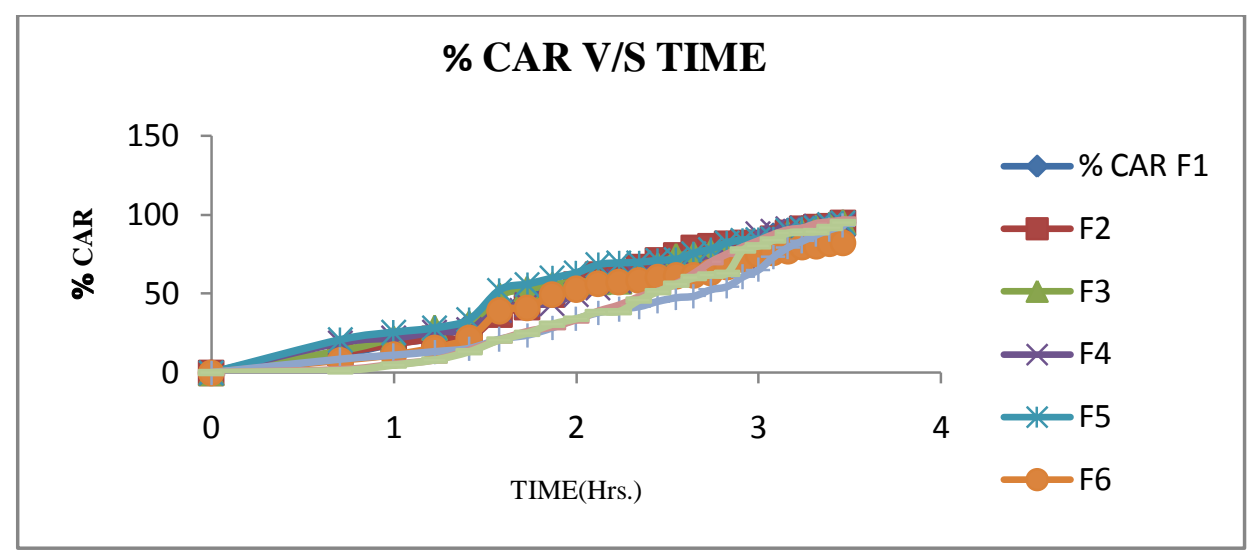

Figure 3: in-vitro drug release profile of salbutamol sulphate F1 to F9

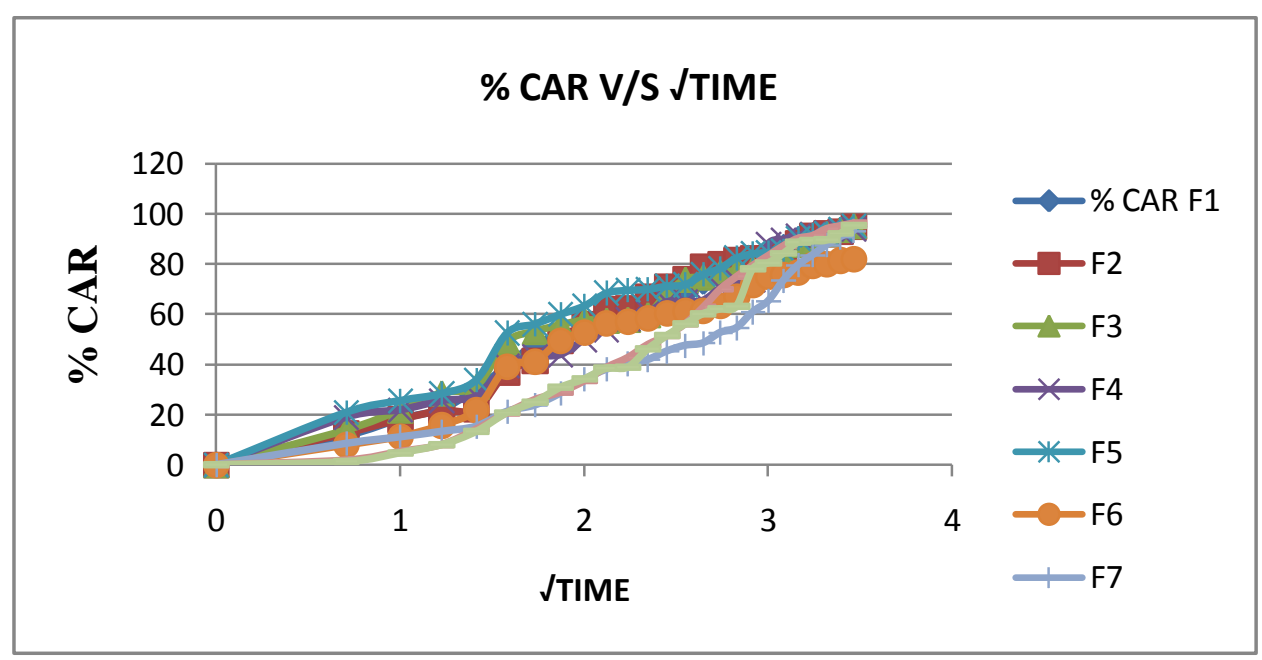

Figure no. 4: Shows Drug Release pattern of Formulation F1 to F9 followed Huguchi model

Table 5: Comparision study of Cumulative $\%$ of drug release, $\mathrm{R}^{2}$ value, $\mathrm{X}_{120}, \mathrm{t} 50$ and $\mathrm{t} 90$ :

\begin{tabular}{|c|c|c|c|c|c|c|}
\hline $\begin{array}{l}\text { FORMULATION } \\
\text { CODE }\end{array}$ & $\%$ CAR & $\begin{array}{c}\mathrm{R}^{2} \mathrm{~V} \\
\text { ZERO }\end{array}$ & $\begin{array}{l}\text { LUE } \\
\text { Higuchi }\end{array}$ & $\mathrm{X}_{120}$ & $\mathrm{t}_{50}(\mathrm{hrs})$ & $\mathrm{t}_{90}(\mathrm{hrs})$ \\
\hline F1 & 94.72 & 0.942 & 0.986 & 30.18 & 3.5 & 10.5 \\
\hline $\mathrm{F} 2$ & 94.98 & 0.918 & 0.974 & 22.17 & 4.0 & 10.5 \\
\hline F3 & 95.35 & 0.915 & 0.983 & 31.27 & 4.5 & 11.0 \\
\hline $\mathrm{F} 4$ & 93.9 & 0.960 & 0.978 & 27.42 & 2.5 & 10.0 \\
\hline F5 & 95.06 & 0.876 & 0.974 & 33.83 & 4.0 & 10.0 \\
\hline F6 & 81.96 & 0.895 & 0.964 & 21.92 & 7.5 & 12 \\
\hline F7 & 93.28 & 0.915 & 0.987 & 15.37 & 6.0 & 11.5 \\
\hline $\mathrm{F} 8$ & 96.53 & 0.936 & 0.989 & 14.22 & 6.0 & 10.0 \\
\hline F9 & 95.4 & 0.935 & 0.989 & 13.3 & 6.0 & 11.5 \\
\hline
\end{tabular}

Table 6: Shows Korsmeyer-Peppas release kinetics

\begin{tabular}{|l|l|c|}
\hline RELEASE EXPONENT & DRUG TRANSPORT & RATE AS A FUNCTION \\
& MECHANISM & $\mathrm{t}$ TIME \\
\hline 0.5 & Fickian diffusion & $\mathrm{t} \mathrm{n}-1$ \\
$0.5<\mathrm{n}<1.0$ & Anomalous transport & Zero-order release \\
1.0 & Case-II transport & $\mathrm{tn}-1$ \\
Higher than 1.0 & Super Case-II transport & \\
\hline
\end{tabular}


Table 7: Model Fitting analysis for formulation F1 to F9

\begin{tabular}{|c|c|c|}
\hline $\begin{array}{c}\text { FORMULATION } \\
\text { CODE }\end{array}$ & KORSMEYER-PEPPAS & DIFFUSION MECHANISM \\
F & 0.776 & Non-Fickian Anamalous \\
F2 & 0.758 & Non- Fickian Anamalous \\
F3 & 0.730 & Non- Fickian Anamalous \\
F4 & 0.749 & Non- Fickian Anamalous \\
F5 & 0.783 & Non- Fickian Anamalous \\
F6 & 0.534 & Non- Fickian Anamalous \\
F7 & 0.596 & Non- Fickian Anamalous \\
F8 & 0.841 & Non- Fickian Anamalous \\
F9 & 0.752 & Non- Fickian Anamalous \\
\hline
\end{tabular}

\section{DISCUSSION:}

Apparent tablet density of all samples showed below $(1.004 \mathrm{~g} / \mathrm{cm} 3)$ than of gastric fluid. The density decreased due to this expansion and upward force of $\mathrm{CO}_{2}$ gas generation. This plays an important role in ensuring the floating capability of the dosage form. To provide good floating behavior in the stomach, the density of the tablets should be less than that of the gastric contents. The drug release data were fitted to models representing zero order (cumulative amount of drug released vs. time), Higuchi's (cumulative percentage of drug released vs. square root of time), and Korsmeyer's equation (log cumulative percentage of drug released vs. time) kinetics to know the release mechanisms. Among all the formulations F8 and F9 showed $\mathrm{R}^{2}$ value of Higuchi model value nearer to unity as compared to the other formulations. The KorsmeyerPeppas (n) data suggest that all formulation show nonfickian transport. Among those formulations F8 formulation were best fitted with korsmeyer model according to n- value.

\section{REFERENCES:}

1) Nayak K Amit, Maji Ruma, Das Biswarup. Gastroretentive drug delivery systems: A review. Asian Journal of Pharmaceutical and Clinical Research [ISSN 0974-2441]. Jan-Mar 2010; 3(1): $1-10$.

2) Nadigoti Jagadeesh, Shayeda. Floating Drug Delivery Systems. International Journal of Pharmaceutical Sciences and Nanotechnology, Oct-Dec 2009; 2(3): 595-604.

3) Streubel A, Siepmann J, Bodmeier R. Gastroretentive drug delivery system. Expert Opin Drug Deliv 2006; IJRPBS.(2011); 3(2): 217-33.

4) Mayavanshi AV, Gajjar SS. Floating drug delivery systems to increase gastric retention of drugs: A Review. Research J. Pharm. And Tech. [ISSN 0974-3618]. Oct.-Dec. 2008; 1(4): 345-348.

5) Kelly HW, Murphy S, Beta-adrenergic agonists for acute, severe asthma. Ann. Pharmacother, 26, 1992, 81-91.

\section{CONCLUSION:}

Gastro retentive floating tablets of Salbutamol sulphate were prepared using two hydrophilic polymer like ethyl cellulose and HPMC by full $3^{2}$ factorial design by changing the ratio of gas generating agent. Nine formulations were prepared. All those formulation showed good acceptable Pharmacotechnical characterstics and able to float within 3 minutes. Formulation like F4, F8 and F9 show higher floation capabilities as well as more steady state drug release profile. On comparative kinetic modeling study (such as Zero order, Higuchi model and Korsmeyer-Peppas model) it was found that all the formulation follow Higuchi model and correlation (R2) values were near to unity. Among those formulation F8 and F9 showed $\mathrm{R}^{2}$ value of Higuchi model more near as compared to the other formulation. In kormeyer pepas model kinetic study it was found that all formulations follow non- fickian diffusion.

The research entitled and result obtained reveals that the combine effect of floating agent in different ratio was suitable for long floating duration.

6) Ahrens RC, Smith GD. Albuterol: an adrenergic agent for use in the treatment of asthma pharmacology, pharmacokinetics and clinical use. 4, 1984, 105-121.

7) Swarbrick J, Boylon JC, Encyclopedia of Pharmaceutical Technology, 1, 2002, 896.

8) Goldstein DA, Tan YK, Soldin SJ, Pharmacokinetics and absolute bioavailability of salbutamol in healthy adult volunteers, Eur J Clin Pharmacol, 32, 1987, 631-634.

9) Pacifici GM, Giulianetti B, Quilici MC, Salbutamol sulphate in the human liver and duodenal mucosa: interindividual variability, Xenobiotica, 27, 1997, 279-286.

10) Crescioli S, Dal CA, Maestrelli P. Controlled-release theophylline inhibits early morning airway obstruction and hyper-responsiveness in asthmatic subjects, Ann Allergy Asthma Immunol, 77, 1996, 106-110. 